\title{
Responses of vertical soil moisture to rainfall pulses and land uses in a typical loess hilly area, China
}

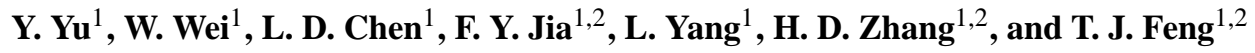 \\ ${ }^{1}$ State Key Laboratory of Urban and Regional Ecology, Research Center for Eco-environmental Sciences, \\ Chinese Academy of Sciences, Beijing 100085, China \\ ${ }^{2}$ University of Chinese Academy of Sciences, Beijing 10049, China \\ Correspondence to: W. Wei (weiwei@rcees.ac.cn)
}

Received: 21 October 2014 - Published in Solid Earth Discuss.: 18 November 2014

Revised: 4 May 2015 - Accepted: 5 May 2015 - Published: 28 May 2015

\begin{abstract}
Soil moisture plays a key role in vegetation restoration and ecosystem stability in arid and semiarid regions. The response of soil moisture to rainfall pulses is an important hydrological process, which is strongly influenced by land use during the implementation of vegetation restoration. In this study, vertical soil moisture variations of woodland (Pinus tabulaeformis), native grassland (Stipa bungeana), shrubland (Hippophea rhamnoides), cropland (Triticum aestivum) and artificial grassland (Onobrychis viciaefolia) in five soil profiles were monitored in a typical loess hilly area during the 2010 growing season. The results demonstrated that rainfall pulses directly affected soil moisture variation. A multi-peak pattern of soil moisture appeared during the growing season, notably in the surface soil layer. Meanwhile, the response of each vegetation type to rainfall was inconsistent, and a time-lag effect before reaching the peak value was detected, following each heavy rainfall event. The response duration of soil moisture, however, varied markedly with the size of rainfall events. Furthermore, higher soil water content was detected in grassland and shrubland. Woodland was characterized by relatively lower soil moisture values throughout the investigation period. Our research suggests that vegetation restoration efforts should give priority to grassland and shrubland at the research site. We suggest that more studies should be focused on the characteristics of community structure and spatial vegetation distribution on soil moisture dynamics, particularly within the grass and shrub ecosystems.
\end{abstract}

\section{Introduction}

Soil moisture is widely recognized as a key factor influencing the success of vegetation restoration and rehabilitation efforts in the semi-arid regions (Chen et al., 2007; FernándezGálvez et al., 2006; Legates et al., 2011; Porporato et al., 2002; Zhao et al., 2013). Meanwhile, it also plays a critical role on the soil improvements (Gabarrón-Galeote, 2013; de Melo Carvalho et al., 2014; Wu et al., 2014; Kaleeem Abbasi et al., 2015; Sadeghi et al., 2015). Consequently, it is imperative to survey the relationship among the water, soil and vegetation interactions, and to explore the synergy effect and feedback mechanisms of the responses of vegetation to precipitation (Cerdà, 1995; Ziadat and Taimeh, 2013; Santos et al., 2013; Hewelke et al., 2014; Gao et al., 2014; Zhu et al., 2014). It follows that soil moisture is the core element functioning as a "cohesive tie" between vegetation and precipitation.

The receipt of water through precipitation is one of the primary factors controlling vegetation dynamics and net primary production in a specific territory. Pulsed rainfall regimes, in turn, affect and control belowground processes via soil wet-dry cycles (Austin et al., 2004). In the semiarid ecosystem, soil wet-dry cycles are influenced by various aspects, such as soil properties, land cover, microlandforms and meteorological environments (Lozano-García et al., 2011; Legates et al., 2011; Yang et al., 2012). In addition, soil moisture dynamics are closely related to infiltration, evaporation, up take of water by roots and as a regulator controlling runoff between different organisms. Typically, soil moisture depends heavily on precipitation pat- 
terns (Koster et al., 2004; Weltzin and Tissue, 2003). In general, with regards to soil moisture responses to precipitation pulse, large precipitation event leads to significant soil moisture pulsation, whereas small rainfall events result in shallow water infiltration (Schwinning and Sala, 2004). Previous research revealed that soil water infiltrated continuously after surface soil was saturated when rainfall events exceeded $5.0 \mathrm{~mm}$, which supplements the root layer effectively (Cerdà, 1997, 1999; Wei et al., 2008). Water balance and the hydrological cycle have always been the critical issues in vegetation restoration in water-controlled ecosystems. Therefore, effectively managing water resources and selecting appropriate vegetation types under limited rainfall conditions are the main tasks of re-vegetation projects in such areas.

The Loess Plateau of China is situated in the upper and middle reaches of the Yellow River. It is a transitional zone between the humid monsoon climate in the southeast and the dry climate in the northwest (Shi and Shao, 2000). Severe water erosion in this region has led to widespread environmental degradation. In order to improve the local ecoenvironment and prevent soil and water loss, the "Grain for Green" project was carried out by the central government in 1999. Most of the sloping croplands were converted to artificial forests and shrublands, and some farmlands were allowed to go fallow as grasslands. Similar as other semiarid ecosystems over the world, land use change can result in soil nutrient levels and property variation (Batjes, 2014; Qadir et al., 2013; Lozano-García and Parras-Alcántara, 2013; Fernández-Romero et al., 2014; Parras-Alcántara and Lozano-García, 2014), even caused significant responses in hydrological function (Bizoza, 2014; Opolot, 2014; Wei et al., 2007; Liu et al., 2012). Additionally, this area has a unique environment in terms of vegetation survival hilly and gully, with intensive and extensive soil erosion and little, but centralized precipitation. Fast growing trees and shrubs have been introduced extensively in this region. Due to water restriction, however, the plants initially grew well but degraded over time, inducing severe soil desiccation (Li, 2001). Small aged trees with heights of 3-5 m were widely distributed and led to low ecosystem productivity. It is necessary to select the suitable vegetation patterns in terms of soil water balance. Therefore, understanding the response of vegetation to soil moisture dynamics is essential for optimizing vegetation structures and achieving the long-term sustainability of ecosystem restoration.

Several studies have demonstrated the effects of vegetation restoration on hydrological processes in the Loess Plateau and other similar regions around the world (Chen et al., 2010; Fu et al., 2013; Qiu et al., 2001; Shangguan and Zheng, 2006; Yang et al., 2014). For example, Chen (2008a) concluded that water hardly reached soil below $200 \mathrm{~cm}$ on the Loess Plateau, both under natural and simulated rainfall conditions. Li (2001) explored how soil infiltration impacted by rainfall events could reach $100-300 \mathrm{~cm}$, with an average of $200 \mathrm{~cm}$. Liu et al (2010) found that shrubs were more adapted to an-

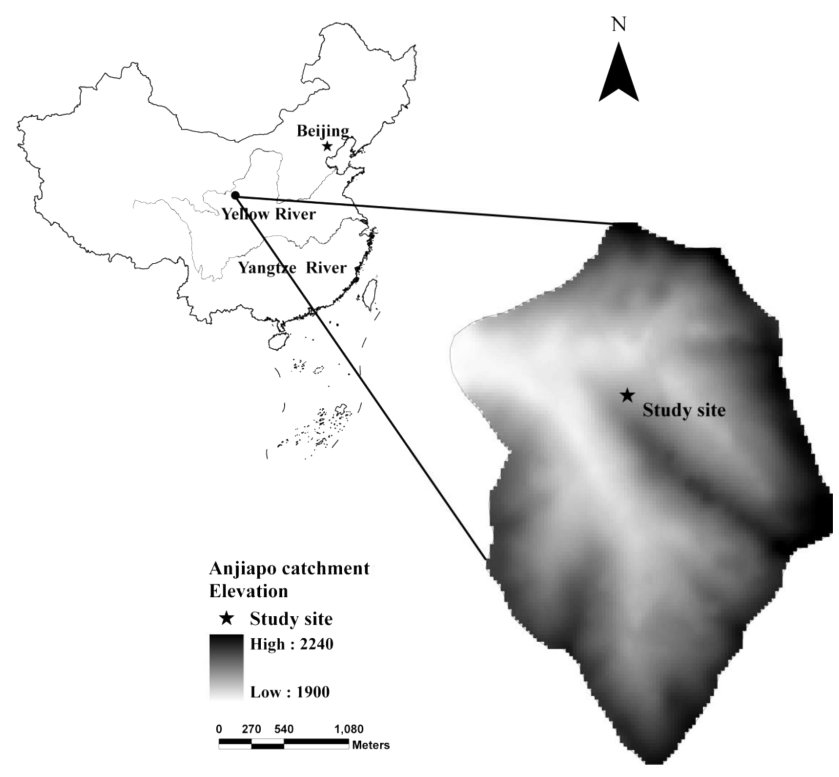

Figure 1. Location of the study site (Anjiapo catchment).

nual rainfall variation than grasses. Nevertheless, it is difficult to make generalizations concerning rainfall pulses and soil moisture dynamics. For instance, a series of small rainfall events is not equal to the same amount of rainfall occurring as a single event, which may lead to greater infiltration and runoff (Schwinning and Sala, 2004). In a way, rainfall distribution coupled with land uses, determines variations of soil moisture for different vegetation types. In addition, many studies have focused on soil desiccation and investigated the excessive depletion of deep soil layers by artificial plants under long-term inadequate rainfall supply on the Loess Plateau (Chen et al., 2008b; Wang et al., 2004, 2011). However, an understanding of the deep mechanisms regarding the responses of soil moisture variation to rainfall pulses and land use remains incomplete. The major purpose of this paper, therefore, is to determine the response of soil moisture variations to rainfall pulses by in situ consecutive monitoring of five typical vegetation types in the loess hilly area of China, including artificial grassland, cropland, shrubland, woodland and native grassland both during and after each rainfall pulse at the plot scale.

\section{Materials and methods}

\subsection{Study site}

The study was conducted in the Anjiapo catchment, at the Dingxi Institute of Soil and Water Conservation Experimental Station $\left(35^{\circ} 33^{\prime}-35^{\circ} 35^{\prime} \mathrm{N}, 104^{\circ} 38^{\prime}-104^{\circ} 41^{\prime} \mathrm{E}\right)$, which belongs to the Chinese Soil and Water Conservation Monitoring Network. The study site represents a typical hilly region with gullies and elevations ranging from 1900 to $2240 \mathrm{~m}$ 
(Fig. 1). The area of experimentation is located in a semi-arid temperate zone with an annual mean precipitation of $427 \mathrm{~mm}$ (1958-2010), more than $80 \%$ of which falls from May to September. The mean annual temperature and, daily maximum and minimum temperatures are $6.3,34.3^{\circ} \mathrm{C}$ in July, and $-27.1^{\circ} \mathrm{C}$ in January, respectively. The mean annual potential transpiration is $1510 \mathrm{~mm}$. The mean length of the frost-free season is 141 days (Wei et al., 2014).

The soil at the study site is of the Calcic Cambisol group in the IUSS-ISRIC-FAO classification system (IUSSISRIC-FAO, 2006). It exhibits a texture composed of $50 \%$ silt $(0.01-0.05 \mathrm{~mm}), 39 \%$ clay $(<0.01 \mathrm{~mm})$ and $11 \%$ sand $(>0.05 \mathrm{~mm})$. The soil field capacity and organic matter at the study site are at $18-24 \%$ and $0.4-1.3 \%$, respectively. The soil bulk density ranges from 1.09 to $1.36 \mathrm{~g} \mathrm{~cm}^{-3}$ in the first $2 \mathrm{~m}$ of depth, and the soil thickness varies from 40 to $60 \mathrm{~m}$ in the same region (Chen et al., 2007; Wei et al., 2014).

With the implementation of the Grain for Green project, Chinese pine (Pinus tabulaeformis Carr.), oriental arborvitae (Platycladus orientalis), purple alfalfa (Medicago sativa), sea buckthorn (Hipporhae rhamnoides L.) and littleleaf peashrub (Caragana microphylla) were widely planted. At present, land-cover patterns in the study area include abandoned cropland, arable land, sloping cropland, native grassland, artificial grassland, shrubland and tree plantations. The main crops are millet (Panicum miliaceum), spring wheat (Triticum aestivum L.), potatoes (Solanum tuberosum L.), soybeans (Vigna angularis), sorghum (Sorghum spp.), and major grasses include Stipa brevifora, Stipa bungeana and Thymis mongolicus.

\subsection{Experimental design and field installation}

A total of 20 experimental plots were distributed on the hill slopes between 10 and $20^{\circ}$, on which rain-fed crops (e.g., wheat, millet and potatoes) had been grown before the plots were constructed. Sample plots used for shrubland and pine woodland were $10 \mathrm{~m} \times 10 \mathrm{~m}$ in size, and $10 \mathrm{~m} \times 5 \mathrm{~m}$ in size, for the sloping cropland, artificial grassland and native grassland. The pine and sea buckthorn were planted in 1978, while the artificial grassland was planted when the plots were constructed in 1986 . Cement ridges $30 \mathrm{~cm}$ above the ground were constructed at the plot borders, while an $\mathrm{H}$-flume was used to measure the surface runoff at the outlet of each plot (Fig. S1 in the Supplement). Four replications were investigated for each vegetation pattern.

Pine trees were planted in the woodland plots with a mean density of $3.0 \mathrm{~m} \times 1.5 \mathrm{~m}$. Little grass was scattered on the surface of the plots. The shrubland plot was dominated by sea buckthorn (Hippophea rhamnoides), with $1 \mathrm{~m}$ distance between rows. During the growing season, dense grasses and thick litter provided a closed cover. For the research, plant residues were kept in the plots. S. bungeana, a dominant native species was sown in the semi-natural grassland plots. In the cropland, T. aestivum was sown in April and harvested manually at the end of July or the beginning of August, and the plant residues were kept in the plots. Additionally, the artificial grassland plots were covered with Sainfoin (Onobrychis viciaefolia) with a height of approximately $0.50 \mathrm{~m}$, which was also harvested manually for livestock.

A total of 10 soil moisture and temperature smart sensors were installed in every plot at 5 depths below the ground. Both the soil moisture and temperature levels of $0-100 \mathrm{~cm}$ profiles were measured for every $20 \mathrm{~cm}$ of depth from May to September (the growing season) of 2010. A pit of appropriate width was dug to allow the probes to be inserted into the soil profile of each plot. Then, the probes were inserted into the unaltered side of the pit and were fixed horizontally in the direction of the maximum slope of each plot. The pits were refilled carefully after the probes were inserted into the soil profile. During this process, it was necessary to avoid any perturbations to the greatest extent possible. The completion of the probe took place at the end of 2009, and the monitoring was not begun until the soil settled, approximately three months later. Before the sensors were installed, the soil bulk density of each depth for every plot was measured using the core method (stainless steel cylinders with a volume of $100 \mathrm{~cm}^{3}$ ) with three replications. The total porosity was calculated according the bulk density and specific weight of soil. Meanwhile, a part of collected soil samples were air dried and visible plant material was removed, sieved through a $2 \mathrm{~mm}$ and then a $1 \mathrm{~mm}$ screen for soil nutrients analysis. Soil organic matter (SOM) was determined using the $\mathrm{K}_{2} \mathrm{CrO}_{7}$ titration method. Total nitrogen (TN) was determined by the semi-micro Kjeldahl method, total phosphorus (TP) was analyzed using colorimetry after wet digestion with $\mathrm{H}_{2} \mathrm{SO}_{4}+\mathrm{HClO}_{4}$, available phosphorus was measured using colorimetry after digestion with $3 \%$ ammonium carbonate (Liu et al., 1996). Alkali-hydrolyzable nitrogen (AN) was determined using the method described by the Cornfield (1960).

\subsection{Measurement sensor}

Soil moisture smart sensors with S-SMC-M005 probes were installed to measure the moisture of the soil profile. Meanwhile, 12-bit temperature smart sensors with S-TMB-M006 probes were prepared to monitor the soil profile temperature. Both the soil moisture and temperature smart sensors were designed to work with smart sensor-compatible HOBO data loggers (Decagon Devices Inc., Pullman, WA).The S-SMCM005 soil moisture smart sensor is capable of providing reading outside the standard volumetric water content range with an accuracy of $\pm 1.0 \%$. All the data were recorded every 10 min using HOBO data loggers. At the same time, each natural rainfall event was measured using a tipping-bucket gauge. The meteorological parameters (solar radiation, air temperature and, wind velocity) were collected as $10 \mathrm{~min}$ averages by an automatic weather station installed in the meteorological garden approximately $1000 \mathrm{~m}$ away from the plot. 
Table 1. Soil properties of five vegetation types.

\begin{tabular}{|c|c|c|c|c|c|c|c|c|}
\hline $\begin{array}{l}\text { Vegetation } \\
\text { type }\end{array}$ & $\begin{array}{c}\text { Depth } \\
(\mathrm{cm})\end{array}$ & $\begin{array}{c}\mathrm{SOM} \\
\left(\mathrm{g} \mathrm{kg}^{-1}\right)\end{array}$ & $\begin{array}{c}\mathrm{TN} \\
\left(\mathrm{g} \mathrm{kg}^{-1}\right)\end{array}$ & $\begin{array}{c}\mathrm{TP} \\
\left(\mathrm{g} \mathrm{kg}^{-1}\right)\end{array}$ & $\begin{array}{c}\mathrm{AN} \\
\left(\mathrm{mg} \mathrm{kg}^{-1}\right)\end{array}$ & $\begin{array}{c}\mathrm{AP} \\
\left(\mathrm{mg} \mathrm{kg}^{-1}\right)\end{array}$ & $\begin{array}{c}\mathrm{BD} \\
\left(\mathrm{g} \mathrm{cm}^{-3}\right)\end{array}$ & $\begin{array}{c}\text { Porosity } \\
(\%)\end{array}$ \\
\hline \multirow{5}{*}{$\begin{array}{l}\text { Artificial } \\
\text { grassland }\end{array}$} & $0-20$ & $10.48 \pm 0.24 \mathrm{Ac}$ & $1.04 \pm 0.02 \mathrm{Ac}$ & $0.81 \pm 0.10 \mathrm{Aa}$ & $56.21 \pm 2.30 \mathrm{Ab}$ & $6.56 \pm 0.01 \mathrm{Abc}$ & $1.16 \pm 0.01 \mathrm{Ab}$ & $55.75 \pm 0.41 \mathrm{Ab}$ \\
\hline & $20-40$ & $8.09 \pm 0.08 \mathrm{Bc}$ & $0.83 \pm 0.04 \mathrm{Bd}$ & $0.67 \pm 0.06 \mathrm{Ba}$ & $46.63 \pm 2.05 \mathrm{Ba}$ & $5.42 \pm 0.17 \mathrm{Bc}$ & $1.26 \pm 0.02 \mathrm{Bb}$ & $52.37 \pm 0.60 \mathrm{Bb}$ \\
\hline & $40-60$ & $7.83 \pm 0.11 \mathrm{Cc}$ & $0.71 \pm 0.02 \mathrm{Cc}$ & $0.64 \pm 0.03 \mathrm{Ba}$ & $35.79 \pm 0.85 \mathrm{Ca}$ & $4.77 \pm 0.11 \mathrm{Cc}$ & $1.26 \pm 0.01 \mathrm{Bb}$ & $52.54 \pm 0.43 \mathrm{Bb}$ \\
\hline & $60-80$ & $7.62 \pm 0.04 \mathrm{Dc}$ & $0.63 \pm 0.03 \mathrm{Dc}$ & $0.65 \pm 0.02 \mathrm{Ba}$ & $31.59 \pm 2.16 \mathrm{Da}$ & $4.75 \pm 0.06 \mathrm{Ca}$ & $1.25 \pm 0.01 \mathrm{Ba}$ & $52.20 \pm 0.42 \mathrm{Ba}$ \\
\hline & $80-100$ & $7.61 \pm 0.03 \mathrm{Dc}$ & $0.60 \pm 0.02 \mathrm{Dc}$ & $0.58 \pm 0.02 \mathrm{Ba}$ & $30.11 \pm 0.55 \mathrm{Da}$ & $4.61 \pm 0.16 \mathrm{Ca}$ & $1.27 \pm 0.01 \mathrm{Ba}$ & $52.79 \pm .041 \mathrm{Ba}$ \\
\hline \multirow[t]{5}{*}{ Cropland } & $0-20$ & $9.27 \pm 0.02 \mathrm{Ac}$ & $0.87 \pm 0.04 \mathrm{Ac}$ & $0.66 \pm 0.01 \mathrm{Ab}$ & $44.05 \pm 2.50 \mathrm{Ac}$ & $8.28 \pm 0.05 \mathrm{Ac}$ & $1.16 \pm 0.01 \mathrm{Ab}$ & $55.84 \pm 0.42 \mathrm{Ab}$ \\
\hline & $20-40$ & $8.03 \pm 0.32 \mathrm{Bc}$ & $0.75 \pm 0.02 \mathrm{Bc}$ & $0.67 \pm 0.02 \mathrm{Aa}$ & $35.34 \pm 0.92 \mathrm{Bc}$ & $8.24 \pm 0.04 \mathrm{Ab}$ & $1.23 \pm 0.01 \mathrm{Bab}$ & $53.11 \pm 0.32 \mathrm{Bab}$ \\
\hline & $40-60$ & $7.41 \pm 0.07 \mathrm{Cc}$ & $0.63 \pm 0.03 \mathrm{Cc}$ & $0.60 \pm 0.03 \mathrm{Bb}$ & $31.37 \pm 0.46 \mathrm{Cbc}$ & $8.16 \pm 0.08 \mathrm{Ab}$ & $1.26 \pm 0.01 \mathrm{Cb}$ & $52.46 \pm 0.31 \mathrm{Cb}$ \\
\hline & $60-80$ & $7.22 \pm 0.17 \mathrm{Cc}$ & $0.60 \pm 0.04 \mathrm{Cc}$ & $0.60 \pm 0.01 \mathrm{Bb}$ & $30.71 \pm 0.72 \mathrm{Ca}$ & $7.96 \pm 0.07 \mathrm{Bb}$ & $1.26 \pm 0.01 \mathrm{Ca}$ & $52.45 \pm 0.41 \mathrm{Ca}$ \\
\hline & $80-100$ & $7.21 \pm 0.09 \mathrm{Cc}$ & $0.59 \pm 0.02 \mathrm{Cc}$ & $0.61 \pm 0.05 \mathrm{Ba}$ & $29.01 \pm 0.08 \mathrm{Ca}$ & $7.62 \pm 0.19 \mathrm{Ca}$ & $1.26 \pm 0.01 \mathrm{Ca}$ & $52.37 \pm 0.38 \mathrm{Ca}$ \\
\hline \multirow[t]{5}{*}{ Shrubland } & $0-20$ & $25.34 \pm 0.86 \mathrm{Aa}$ & $1.66 \pm 0.03 \mathrm{Aa}$ & $0.74 \pm 0.04 \mathrm{Aab}$ & $70.09 \pm 3.59 \mathrm{Aa}$ & $12.01 \pm 0.06 \mathrm{Aa}$ & $1.13 \pm 0.02 \mathrm{Aa}$ & $57.08 \pm 1.14 \mathrm{Aa}$ \\
\hline & $20-40$ & $20.65 \pm 0.55 \mathrm{Ba}$ & $1.35 \pm 0.04 \mathrm{Ba}$ & $0.67 \pm 0.02 \mathrm{Aa}$ & $49.89 \pm 3.69 \mathrm{Ba}$ & $9.78 \pm 0.08 \mathrm{Ba}$ & $1.20 \pm 0.01 \mathrm{Ba}$ & $53.86 \pm 0.43 \mathrm{Ba}$ \\
\hline & $40-60$ & $20.14 \pm 0.93 \mathrm{Ba}$ & $1.28 \pm 0.02 \mathrm{Ba}$ & $0.63 \pm 0.01 \mathrm{Bab}$ & $32.12 \pm 2.49 \mathrm{Cb}$ & $9.17 \pm 0.14 \mathrm{Ca}$ & $1.23 \pm 0.01 \mathrm{Ca}$ & $52.70 \pm 0.47 \mathrm{Ca}$ \\
\hline & $60-80$ & $19.65 \pm 0.35 \mathrm{Ba}$ & $1.24 \pm 0.04 \mathrm{Ba}$ & $0.60 \pm 0.10 \mathrm{BCb}$ & $23.69 \pm 0.23 \mathrm{Db}$ & $9.16 \pm 0.06 \mathrm{Ca}$ & $1.24 \pm 0.01 \mathrm{Ca}$ & $52.46 \pm 0.42 \mathrm{Ca}$ \\
\hline & $80-100$ & $10.76 \pm 0.16 \mathrm{Ca}$ & $1.36 \pm 0.11 \mathrm{Ba}$ & $0.58 \pm 0.02 \mathrm{Ca}$ & $21.81 \pm 1.70 \mathrm{Db}$ & $9.06 \pm 0.03 \mathrm{Ca}$ & $1.25 \pm 0.01 \mathrm{Ca}$ & $52.37 \pm 0.38 \mathrm{Ca}$ \\
\hline \multirow[t]{5}{*}{ Woodland } & $0-20$ & $18.31 \pm 0.06 \mathrm{Ab}$ & $1.23 \pm 0.03 \mathrm{Ab}$ & $1.14 \pm 0.07 \mathrm{Ab}$ & $39.42 \pm 0.13 \mathrm{Ac}$ & $6.01 \pm 0.10 \mathrm{Ac}$ & $1.19 \pm 0.02 \mathrm{Ab}$ & $54.68 \pm 0.50 \mathrm{Aab}$ \\
\hline & $20-40$ & $14.37 \pm 0.35 \mathrm{Bb}$ & $1.14 \pm 0.03 \mathrm{Bb}$ & $0.84 \pm 0.09 \mathrm{Ba}$ & $17.25 \pm 0.25 \mathrm{Bb}$ & $5.06 \pm 0.49 \mathrm{Bd}$ & $1.24 \pm 0.01 \mathrm{Bb}$ & $52.46 \pm 0.42 \mathrm{Bb}$ \\
\hline & $40-60$ & $12.26 \pm 0.07 \mathrm{Cb}$ & $1.15 \pm 0.03 \mathrm{Bb}$ & $0.76 \pm 0.02 \mathrm{BCab}$ & $15.29 \pm 0.26 \mathrm{Cbc}$ & $4.70 \pm 0.11 \mathrm{Cd}$ & $1.26 \pm 0.01 \mathrm{Cb}$ & $51.30 \pm 0.41 \mathrm{Cb}$ \\
\hline & $60-80$ & $11.69 \pm 0.33 \mathrm{Db}$ & $1.07 \pm 0.04 \mathrm{Cb}$ & $0.75 \pm 0.03 \mathrm{BCb}$ & $11.26 \pm 0.22 \mathrm{Db}$ & $4.62 \pm 0.04 \mathrm{Cc}$ & $1.30 \pm 0.01 \mathrm{Ca}$ & $50.55 \pm 0.43 \mathrm{Ca}$ \\
\hline & $80-100$ & $11.34 \pm 0.14 \mathrm{Dab}$ & $1.06 \pm 0.01 \mathrm{Cb}$ & $0.53 \pm 0.01 \mathrm{Ca}$ & $10.62 \pm 0.27 \mathrm{Db}$ & $4.56 \pm 0.02 \mathrm{Ca}$ & $1.28 \pm 0.01 \mathrm{Ca}$ & $51.63 \pm 0.32 \mathrm{Ca}$ \\
\hline \multirow{5}{*}{$\begin{array}{l}\text { Native } \\
\text { grassland }\end{array}$} & $0-20$ & $21.89 \pm 1.30 \mathrm{Ab}$ & $1.22 \pm 0.05 \mathrm{Ab}$ & $0.67 \pm 0.03 \mathrm{Ab}$ & $45.93 \pm 1.12 \mathrm{Ac}$ & $4.65 \pm 003 \mathrm{Ac}$ & $1.15 \pm 0.02 \mathrm{Aab}$ & $56.08 \pm 0.56 \mathrm{Aab}$ \\
\hline & $20-40$ & $16.61 \pm 0.35 \mathrm{Bb}$ & $1.06 \pm 0.02 \mathrm{Bb}$ & $0.64 \pm 0.01 \mathrm{Ba}$ & $39.38 \pm 1.60 \mathrm{Bb}$ & $4.37 \pm 0.02 \mathrm{Bd}$ & $1.24 \pm 0.02 \mathrm{Bb}$ & $52.78 \pm 0.55 \mathrm{Bb}$ \\
\hline & $40-60$ & $12.23 \pm 0.06 \mathrm{Cb}$ & $1.03 \pm 0.12 \mathrm{BCb}$ & $0.63 \pm 0.02 \mathrm{BCab}$ & $28.93 \pm 2.15 \mathrm{Cc}$ & $4.01 \pm 0.03 \mathrm{BCd}$ & $1.25 \pm 0.01 \mathrm{Bb}$ & $52.86 \pm 0.42 \mathrm{Bb}$ \\
\hline & $60-80$ & $11.40 \pm 0.51 \mathrm{CDb}$ & $1.04 \pm 0.04 \mathrm{BCb}$ & $0.62 \pm 0.02 \mathrm{BCb}$ & $22.59 \pm 1.24 \mathrm{Db}$ & $3.92 \pm 0.08 \mathrm{Cc}$ & $1.25 \pm 0.01 \mathrm{Ba}$ & $52.70 \pm 0.47 \mathrm{Ba}$ \\
\hline & $80-100$ & $10.45 \pm 0.25 \mathrm{Db}$ & $1.04 \pm 0.01 \mathrm{Cb}$ & $0.59 \pm 0.05 \mathrm{Ca}$ & $23.94 \pm 0.48 \mathrm{Db}$ & $3.91 \pm 0.07 \mathrm{Ca}$ & $1.25 \pm 0.01 \mathrm{Ba}$ & $52.69 \pm 0.42 \mathrm{Ba}$ \\
\hline
\end{tabular}

Data in the figure were mean and standard deviation (SD). Different uppercase letters indicate significance in different soil depth; different lowercase letters indicate significant differences in different vegetation types $(P<0.05)$. Abbreviations: soil organic matter (SOM), total nitrogen (TN), total phosphorous (TP), alkali-hydrolyzable nitrogen $(\mathrm{AN})$, available phosphorus (AP), bulk density (BD).

\subsection{Statistical analysis}

Since the experiments were carried out at the plot level, the environmental factors exhibited homogeneous characteristics for different vegetation types. Therefore, the temporal dynamics of soil moisture and its relationship with vegetation type were analyzed. The average soil moisture of specific vegetation types and depths were calculated using Eq. (1), as follows (Chen et al., 2007):

$S_{i j}=\frac{1}{4 \times n} \sum_{m=1}^{4} \sum_{k=1}^{n} S_{i j m k}$,

where $S_{i j}$ is the volumetric soil water content $\left(\mathrm{cm}^{3} \mathrm{~cm}^{-3}\right)$ of $i$ th vegetation pattern at $j$ th depth; $S_{i j m k}$ is the actual value of volumetric soil water content $\left(\mathrm{cm}^{3} \mathrm{~cm}^{-3}\right)$ measurement each time; $n$ is the total number of volumetric soil water content monitoring from May to September in 2010 and number 4 is the replications.

At the same time, we assumed that the volumetric soil water content was measured with probes precisely matching different layers in the soil profile (Wang et al., 2012). According to the principle of soil water balance, the cumulative water loss can therefore be calculated by Eq. (2), as follows:

$\mathrm{WL}_{i}=\left(S_{b i}-S_{e i}\right) \times D$, where $\mathrm{WL}_{i}$ is the cumulative loss water $(\mathrm{mm})$ of $i$ th vegetation type; $S_{\mathrm{b} i}$ is the initial volumetric soil water content $\left(\mathrm{cm}^{3} \mathrm{~cm}^{-3}\right)$ of $i$ th vegetation type and $S_{\mathrm{e} i}$ is the volumetric soil water content $\left(\mathrm{cm}^{3} \mathrm{~cm}^{-3}\right)$ of $i$ th vegetation type at the end stage. $D$ is the depth $(\mathrm{mm})$ of the measurement.

Data on soil moisture and soil temperature were analyzed to provide mean and standard deviation (SD) for each variable measured at every depth in each plot. Analysis of variance was performed using the MIXED procedure in SAS, which computes Wald-type F-statistics using generalized least squares (GLSEs) based on restricted maximum likelihood estimates of the variance components (Littell et al., 1996). In the case of significant differences in the Wald-Fstatistic at $P<0.05$, treatment means were compared using an LSD (least significant difference) test. All statistical analyses were conducted using the SAS 9.2 software package (SAS, 2002).

\section{Results}

\subsection{Soil properties}

Soil physicochemical properties varied among vegetation types (Table 1). SOM, TN, AN, AP values from shrubland were statistically higher than those of other vegetation types. 


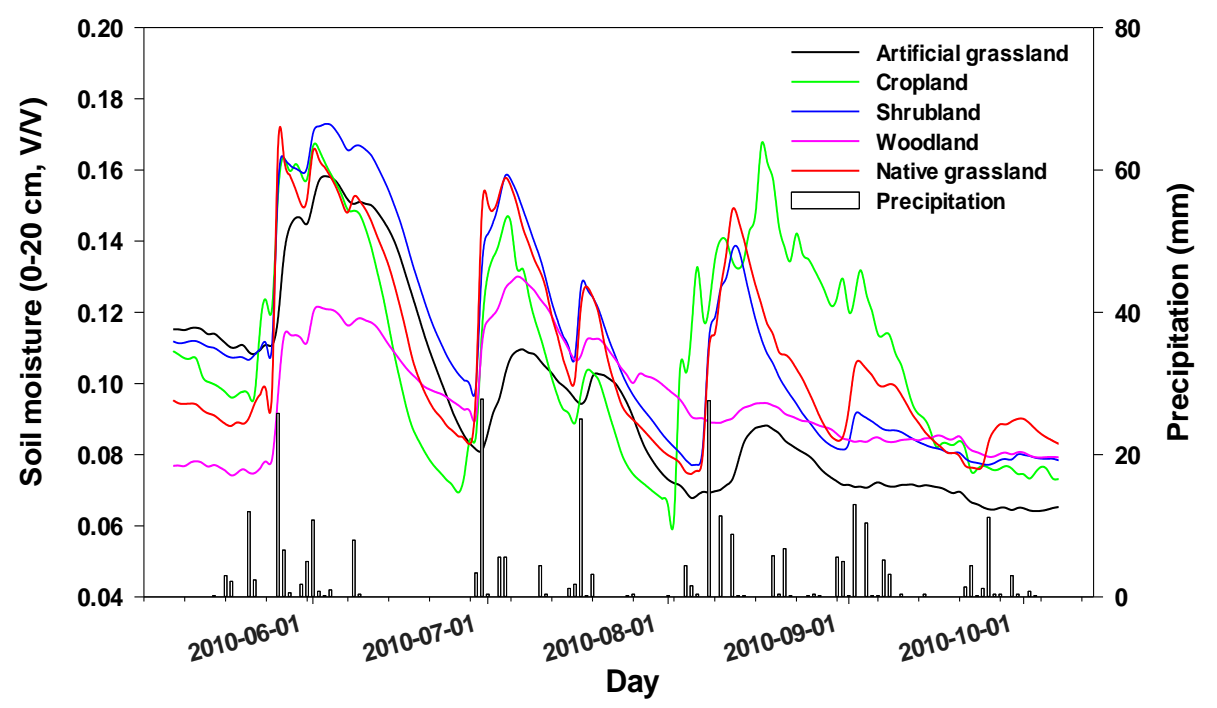

Figure 2. Characteristics of soil moisture variation in the $0-20 \mathrm{~cm}$ of five vegetation types.

The values of SOM and TN of native grassland were higher than that of artificial grassland, while AN and AP values of native grassland were lower than that of artificial grassland. SOM of the woodland was lower than the native grassland but higher than artificial grassland. In short, the level of SOM of the five types was in the following order, Shrubland $\left(19.31 \mathrm{~g} \mathrm{~kg}^{-1}\right)>$ native grassland $\left(14.51 \mathrm{~g} \mathrm{~kg}^{-1}\right)>$ woodland $\left(13.59 \mathrm{~g} \mathrm{~kg}^{-1}\right)>$ artificial grassland $\left(8.31 \mathrm{~g} \mathrm{~kg}^{-1}\right)>$ cropland $\left(7.83 \mathrm{~g} \mathrm{~kg}^{-1}\right)$. In addition, shrubland had the lowest BD values compared with other vegetation types. In the surface layer, although there was no significant difference of the $\mathrm{BD}$ value between shrubland $\left(1.13 \mathrm{~g} \mathrm{~cm}^{-3}\right)$ and native grassland $\left(1.15 \mathrm{~g} \mathrm{~cm}^{-3}\right)$, the BD value of shrubland exhibited significant lower than artificial grassland $\left(1.16 \mathrm{~g} \mathrm{~cm}^{-3}\right)$, cropland $\left(1.16 \mathrm{~g} \mathrm{~cm}^{-3}\right)$ and woodland $\left(1.19 \mathrm{~g} \mathrm{~cm}^{-3}\right)(P<0.05)$. However, there explored non-significant difference between native grassland and artificial grassland. BD values were similar below a depth of $40 \mathrm{~cm}$, and all were significantly higher than the surface layer in each plot $(P<0.05)$. In general, shrubland has the lowest $\mathrm{BD}$ with highest total porosity. The level of $\mathrm{BD}$ values of the five vegetation types was in the following order: shrubland $<$ native grassland $<$ cropland $<$ artificial grassland $<$ woodland.

\subsection{Rainfall features in the study area}

As shown in Fig. 2, 76 rainfall events occurred during observation period (1 April-31 October) in 2010, with the cumulative rainfall reaching $322.6 \mathrm{~mm}$. The total rainfall for the growing season (1 May-30 September) was $292.8 \mathrm{~mm}$, accounting for $90.1 \%$ of the observation period. The maximum and minimum daily rainfall was 27.8 and $0.2 \mathrm{~mm}$, respectively. Daily rainfall greater than $25 \mathrm{~mm}$ occurred on four occasions: 25 May $(25.8 \mathrm{~mm}), 29$ June $(27.8 \mathrm{~mm}), 16$ July $(25.0 \mathrm{~mm})$ and 7 August $(27.8 \mathrm{~mm})$, respectively, account- ing for $32.9 \%$ of the total rainfall for the growing season. On six occasions, daily rainfall was greater than $10 \mathrm{~mm}$, and 11 times it was between 10 and $5 \mathrm{~mm}$. On 47, daily rainfall events less than $5 \mathrm{~mm}$ occurred during the growing season. Overall, the study area mainly experienced small rainfall events, with heavy rainfall events occurring less frequently. The total rainfall during the growing season, however, was strongly influenced by the heavy rainfall regimes. Therefore, the rainfall characteristics of the experimental site were typical pulse rainfall events of the semi-arid zone.

\subsection{Seasonal variation pattern and pulse of soil moisture}

The trends of soil moisture variation of the five vegetation types exhibited a similar seasonal variation during the growing season, particularly in the $0-20 \mathrm{~cm}$ soil layer (Figs. 2 and 3 ). Native grassland had the highest soil moisture (5 June) while the cropland had the lowest (2 August). In the top soil layer $(0-20 \mathrm{~cm})$, the seasonal changes in soil moisture were apparent, and each vegetation type had four peak values based on time under rainfall conditions. However, there was no consistency in the way each vegetation type to reached its peak value. Furthermore, three-peak, double-peak and single-peak phenomena were obviously present during the growing season (Fig. 2), particularly after heavy rainfall $(>25 \mathrm{~mm})$ at $0-20 \mathrm{~cm}$. Additionally, a hysteresis effect was apparent following a heavy rainfall event when the peak value was reached. For instance, during the investigation period from 25 May to 28 June, after the heavy rainfall pulse (28 May, $25.8 \mathrm{~mm}$ ) occurred, different types of vegetation exhibited three peak-value phenomena in the top soil layer, with the three peak times for the shrubland taking place on 27 May, 4 June and 10 June; for the crop land on 27 May, 1 June and 8 June; the artificial grassland on 29 May, 3 June 

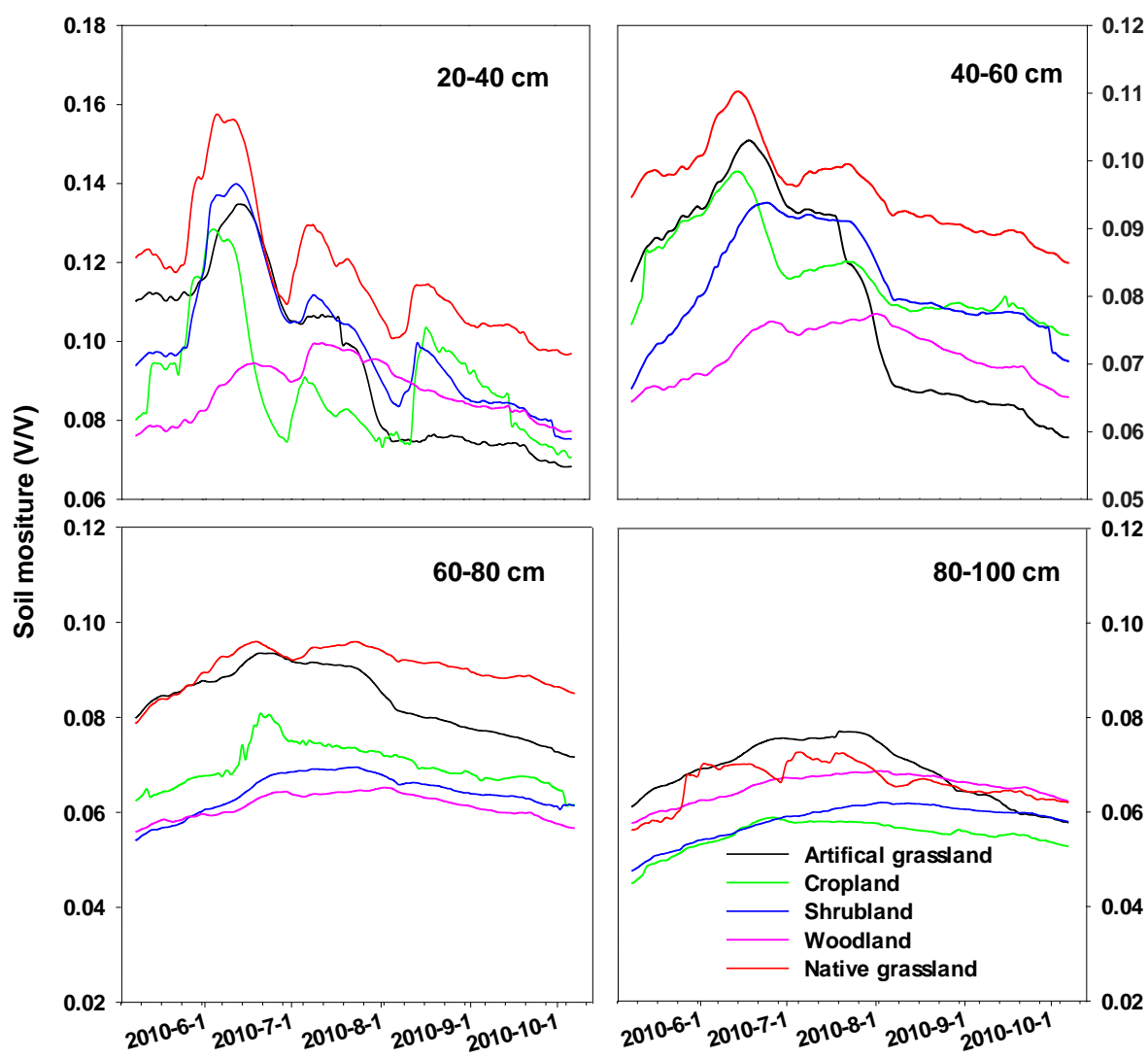

Date

Figure 3. Characteristics of soil moisture variation in the $20-100 \mathrm{~cm}$ zone of five vegetation types.

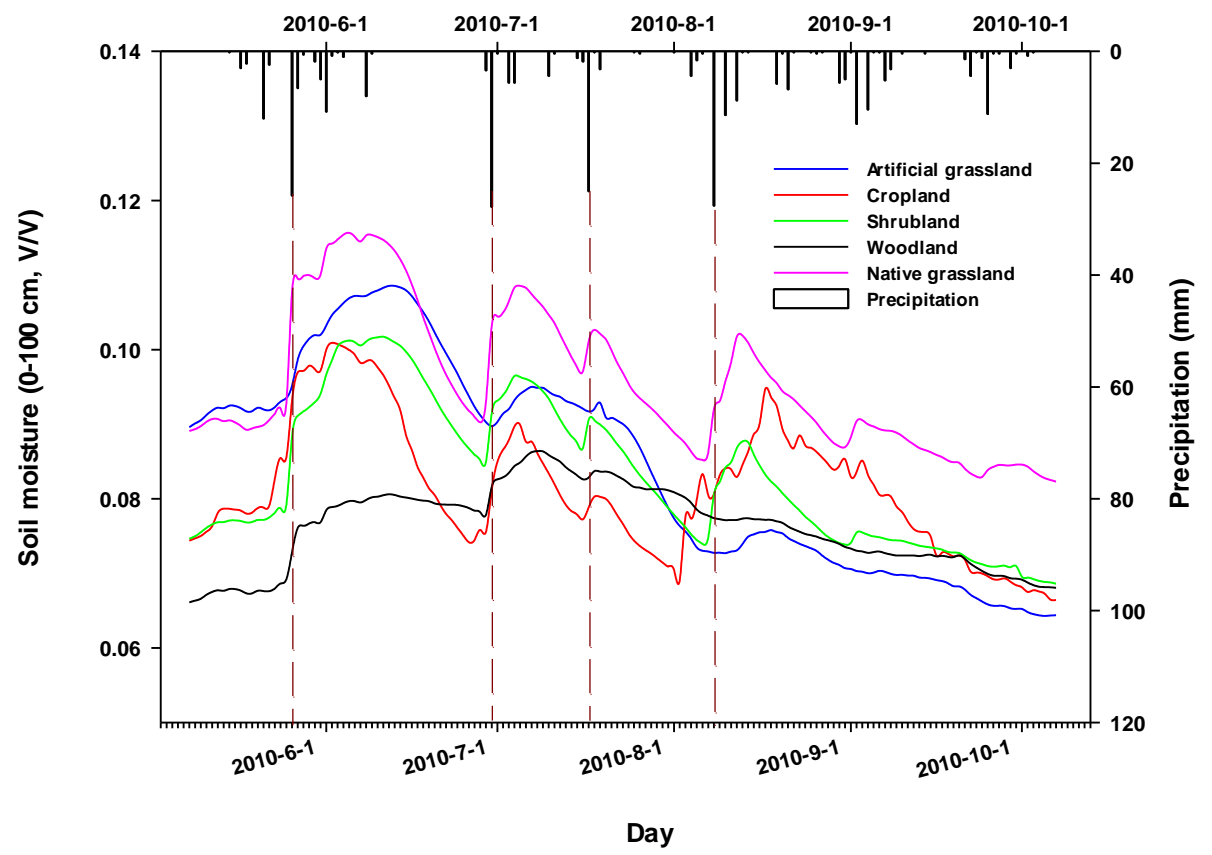

Figure 4. Characteristics of soil moisture variation during the growing season of five types of vegetation. 
Table 2. Soil moisture variation of five vegetation types during the growing season.

\begin{tabular}{|c|c|c|c|c|c|c|}
\hline \multirow[b]{2}{*}{ Months } & \multirow[b]{2}{*}{ Depth/cm } & \multicolumn{5}{|c|}{ Volumetric water content $\left(\mathrm{cm}^{3} \mathrm{~cm}^{-3}\right)$} \\
\hline & & Artificial grassland & Cropland & Shrubland & Woodland & Native grassland \\
\hline \multirow[t]{6}{*}{ May } & $0-20$ & $0.12 \pm 0.02 \mathrm{Aa}$ & $0.12 \pm 0.01 \mathrm{Aa}$ & $0.12 \pm 0.05 \mathrm{Aa}$ & $0.08 \pm 0.04 \mathrm{Aa}$ & $0.11 \pm 0.03 \mathrm{Aa}$ \\
\hline & $20-40$ & $0.11 \pm 0.02 \mathrm{Aa}$ & $0.10 \pm 0.02 \mathrm{ABa}$ & $0.10 \pm 0.04 \mathrm{ABa}$ & $0.08 \pm 0.03 \mathrm{Aa}$ & $0.12 \pm 0.04 \mathrm{Aa}$ \\
\hline & $40-60$ & $0.09 \pm 0.03 \mathrm{Aa}$ & $0.09 \pm 0.02 \mathrm{ABCa}$ & $0.07 \pm 0.02 \mathrm{ABa}$ & $0.07 \pm 0.04 \mathrm{Aa}$ & $0.10 \pm 0.04 \mathrm{Aa}$ \\
\hline & $60-80$ & $0.08 \pm 0.03 \mathrm{Aa}$ & $0.07 \pm 0.03 \mathrm{BCa}$ & $0.06 \pm 0.02 \mathrm{ABa}$ & $0.06 \pm 0.02 \mathrm{Aa}$ & $0.08 \pm 0.04 \mathrm{Aa}$ \\
\hline & $80-100$ & $0.07 \pm 0.03 \mathrm{Aa}$ & $0.05 \pm 0.02 \mathrm{Ca}$ & $0.06 \pm 0.03 \mathrm{Ba}$ & $0.06 \pm 0.03 \mathrm{Aa}$ & $0.06 \pm 0.03 \mathrm{Aa}$ \\
\hline & $0-100$ & $0.09 \pm 0.03 \mathrm{a}$ & $0.08 \pm 0.03 \mathrm{a}$ & $0.08 \pm 0.04 a$ & $0.07 \pm 0.03 \mathrm{a}$ & $0.10 \pm 0.04 \mathrm{a}$ \\
\hline \multirow[t]{6}{*}{ June } & $0-20$ & $0.13 \pm 0.02 \mathrm{Aa}$ & $0.11 \pm 0.01 \mathrm{Aa}$ & $0.14 \pm 0.03 \mathrm{Aa}$ & $0.11 \pm 0.04 \mathrm{Aa}$ & $0.12 \pm 0.03 \mathrm{Aa}$ \\
\hline & $20-40$ & $0.12 \pm 0.02 \mathrm{Aa}$ & $0.10 \pm 0.02 \mathrm{ABa}$ & $0.13 \pm 0.02 \mathrm{Aa}$ & $0.09 \pm 0.03 \mathrm{Aa}$ & $0.14 \pm 0.04 \mathrm{Aa}$ \\
\hline & $40-60$ & $0.10 \pm 0.03 \mathrm{Aa}$ & $0.09 \pm 0.01 \mathrm{ABCa}$ & $0.09 \pm 0.03 \mathrm{Aba}$ & $0.07 \pm 0.04 \mathrm{Aa}$ & $0.10 \pm 0.02 \mathrm{Aa}$ \\
\hline & $60-80$ & $0.09 \pm 0.03 \mathrm{Aa}$ & $0.07 \pm 0.02 \mathrm{BCa}$ & $0.06 \pm 0.02 \mathrm{Ba}$ & $0.06 \pm 0.02 \mathrm{Aa}$ & $0.09 \pm 0.04 \mathrm{Aa}$ \\
\hline & $80-100$ & $0.07 \pm 0.03 \mathrm{Aa}$ & $0.06 \pm 0.02 \mathrm{Ca}$ & $0.06 \pm 0.02 \mathrm{Ba}$ & $0.06 \pm 0.02 \mathrm{Aa}$ & $0.07 \pm 0.02 \mathrm{Aa}$ \\
\hline & $0-100$ & $0.10 \pm 0.03 \mathrm{a}$ & $0.08 \pm 0.02 \mathrm{a}$ & $0.09 \pm 0.03 \mathrm{a}$ & $0.08 \pm 0.03 \mathrm{a}$ & $0.10 \pm 0.04 \mathrm{a}$ \\
\hline \multirow[t]{6}{*}{ July } & $0-20$ & $0.10 \pm 0.02 \mathrm{Aa}$ & $0.10 \pm 0.01 \mathrm{Aa}$ & $0.12 \pm 0.03 \mathrm{Aa}$ & $0.11 \pm 0.04 \mathrm{Aa}$ & $0.12 \pm 0.03 \mathrm{Aa}$ \\
\hline & $20-40$ & $0.10 \pm 0.02 \mathrm{Aa}$ & $0.08 \pm 0.01 \mathrm{ABa}$ & $0.10 \pm 0.02 \mathrm{ABa}$ & $0.10 \pm 0.03 \mathrm{Aa}$ & $0.12 \pm 0.04 \mathrm{Aa}$ \\
\hline & $40-60$ & $0.09 \pm 0.03 \mathrm{Aa}$ & $0.08 \pm 0.01 \mathrm{ABa}$ & $0.09 \pm 0.02 \mathrm{ABa}$ & $0.08 \pm 0.04 \mathrm{Aa}$ & $0.10 \pm 0.02 \mathrm{Aa}$ \\
\hline & $60-80$ & $0.09 \pm 0.04 \mathrm{Aa}$ & $0.07 \pm 0.01 \mathrm{Ba}$ & $0.07 \pm 0.02 \mathrm{Ba}$ & $0.06 \pm 0.02 \mathrm{Aa}$ & $0.09 \pm 0.03 \mathrm{Aa}$ \\
\hline & $80-100$ & $0.08 \pm 0.03 \mathrm{Aa}$ & $0.06 \pm 0.02 \mathrm{Ba}$ & $0.06 \pm 0.02 \mathrm{Ba}$ & $0.07 \pm 0.02 \mathrm{Aa}$ & $0.07 \pm 0.04 \mathrm{Aa}$ \\
\hline & $0-100$ & $0.09 \pm 0.02 \mathrm{a}$ & $0.08 \pm 0.01 \mathrm{a}$ & $0.09 \pm 0.03 \mathrm{a}$ & $0.09 \pm 0.03 \mathrm{a}$ & $0.10 \pm 0.04 \mathrm{a}$ \\
\hline \multirow[t]{6}{*}{ August } & $0-20$ & $0.08 \pm 0.01 \mathrm{Aa}$ & $0.13 \pm 0.05 \mathrm{Aa}$ & $0.10 \pm 0.04 \mathrm{Aa}$ & $0.09 \pm 0.02 \mathrm{Aa}$ & $0.10 \pm 0.04 \mathrm{Aa}$ \\
\hline & $20-40$ & $0.08 \pm 0.01 \mathrm{Aa}$ & $0.09 \pm 0.02 \mathrm{ABa}$ & $0.09 \pm 0.03 \mathrm{Aa}$ & $0.09 \pm 0.01 \mathrm{Aa}$ & $0.10 \pm 0.04 \mathrm{Aa}$ \\
\hline & $40-60$ & $0.07 \pm 0.02 \mathrm{Aa}$ & $0.08 \pm 0.01 \mathrm{ABa}$ & $0.08 \pm 0.02 \mathrm{Aa}$ & $0.07 \pm 0.03 \mathrm{Aa}$ & $0.11 \pm 0.04 \mathrm{Aa}$ \\
\hline & $60-80$ & $0.08 \pm 0.03 \mathrm{Aa}$ & $0.07 \pm 0.01 \mathrm{Ba}$ & $0.07 \pm 0.02 \mathrm{Aa}$ & $0.06 \pm 0.02 \mathrm{Aa}$ & $0.09 \pm 0.03 \mathrm{Aa}$ \\
\hline & $80-100$ & $0.07 \pm 0.03 \mathrm{Aa}$ & $0.06 \pm 0.02 \mathrm{Ba}$ & $0.06 \pm 0.02 \mathrm{Aa}$ & $0.07 \pm 0.02 \mathrm{Aa}$ & $0.07 \pm 0.04 \mathrm{Aa}$ \\
\hline & $0-100$ & $0.07 \pm 0.02 \mathrm{a}$ & $0.09 \pm 0.03 a$ & $0.08 \pm 0.02 \mathrm{a}$ & $0.08 \pm 0.02 \mathrm{a}$ & $0.09 \pm 0.03 \mathrm{a}$ \\
\hline \multirow[t]{6}{*}{ September } & $0-20$ & $0.07 \pm 0.01 \mathrm{Aa}$ & $0.09 \pm 0.02 \mathrm{Aa}$ & $0.08 \pm 0.03 \mathrm{Aa}$ & $0.08 \pm 0.01 \mathrm{Aa}$ & $0.09 \pm 0.03 \mathrm{Aa}$ \\
\hline & $20-40$ & $0.07 \pm 0.01 \mathrm{Aa}$ & $0.08 \pm 0.02 \mathrm{ABa}$ & $0.08 \pm 0.02 \mathrm{Aa}$ & $0.08 \pm 0.01 \mathrm{Aa}$ & $0.10 \pm 0.04 \mathrm{Aa}$ \\
\hline & $40-60$ & $0.07 \pm 0.01 \mathrm{Aa}$ & $0.08 \pm 0.01 \mathrm{ABa}$ & $0.08 \pm 0.02 \mathrm{Aa}$ & $0.07 \pm 0.02 \mathrm{Aa}$ & $0.09 \pm 0.03 \mathrm{Aa}$ \\
\hline & $60-80$ & $0.08 \pm 0.02 \mathrm{Aa}$ & $0.07 \pm 0.01 \mathrm{ABa}$ & $0.06 \pm 0.02 \mathrm{Aa}$ & $0.06 \pm 0.02 \mathrm{Aa}$ & $0.09 \pm 0.03 \mathrm{Aa}$ \\
\hline & $80-100$ & $0.06 \pm 0.02 \mathrm{Aa}$ & $0.05 \pm 0.02 \mathrm{Ba}$ & $0.06 \pm 0.02 \mathrm{Aa}$ & $0.07 \pm 0.01 \mathrm{Aa}$ & $0.06 \pm 0.03 \mathrm{Aa}$ \\
\hline & $0-100$ & $0.07 \pm 0.01 \mathrm{a}$ & $0.08 \pm 0.02 \mathrm{a}$ & $0.07 \pm 0.02 \mathrm{a}$ & $0.07 \pm 0.01 \mathrm{a}$ & $0.09 \pm 0.03 \mathrm{a}$ \\
\hline
\end{tabular}

Values are mean $\pm \mathrm{SD}$ ( $n=4$ for each vegetation type). Different uppercase letters indicate significant differences in different soil depths, different lowercase letters indicate significant differences in different vegetation types $(P<0.05)$.

and 9 June; while the woodland on 26 May, 2 June and 10 June, and the native grassland on 26 May, 1 June and 11 June, respectively. These times, therefore, represented the relatively wetter conditions of the soil. The degree of the soil moisture variation dramatically decreased with increased soil depth.

As shown in Fig. 4, little rainfall occurred from 5 to 14 July, 20 July to 3 August, 22 to 28 August and 8 to 19 September. Meanwhile, no rainfall event happened from 9 to 28 June. During these periods, soil volumetric water content declined continuously. However, the minimum mean value for each vegetation type occurred at different times in various decreasing stages. For instance, during the experimental period from 9 to 28 June, before the second heavy rainfall event happened during the growing season (29 June, $27.8 \mathrm{~mm}$ ), no rainfall was recorded on these days.
The minimum mean value took place on 27 June for the cropland $\left(0.074 \mathrm{~cm}^{3} \mathrm{~cm}^{-3}\right)$, the woodland $\left(0.079 \mathrm{~cm}^{3} \mathrm{~cm}^{-3}\right)$ and the shrubland $\left(0.085 \mathrm{~cm}^{3} \mathrm{~cm}^{-3}\right)$ on 29 June, and the native grassland $\left(0.090 \mathrm{~cm}^{3} \mathrm{~cm}^{-3}\right)$ on 28 June. In contrast, the minimum mean value for the artificial grassland $\left(0.089 \mathrm{~cm}^{3} \mathrm{~cm}^{-3}\right)$ appeared on $30 \mathrm{June}$, after the second heavy rainfall event. These days represented the time at which the drier soil conditions occurred for the various vegetation types. In general, for all of the rainfall regimes during the investigated growing season, largely similar curves with obviously different trends in soil moisture variation were clearly exhibited among the different vegetation types. There was no doubt that the variability in soil moisture depended heavily on precipitation. 


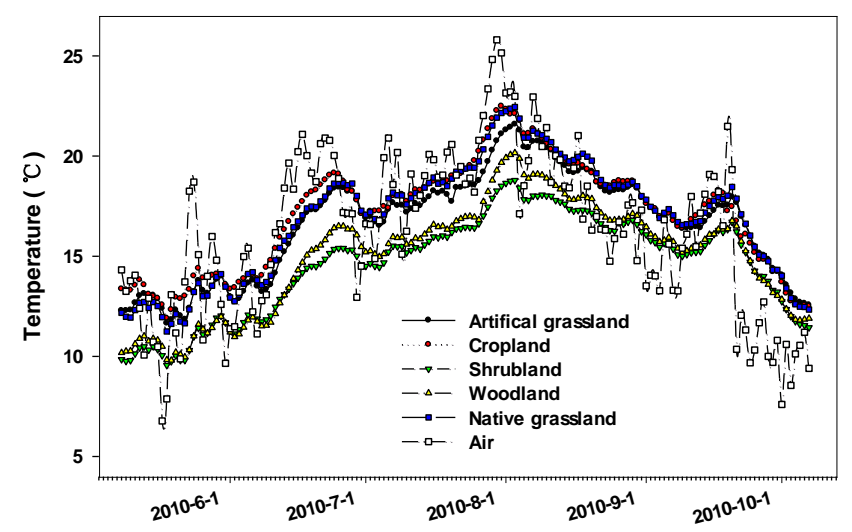

Figure 5. Characteristic of soil temperature variations of five vegetation patterns.

\subsection{Soil moisture and temperature variation of different types of vegetation}

The mean volumetric water contents of the $0-100 \mathrm{~cm}$ soil profile were shown in Table 2. Soil moisture variation was different at different soil depths. There was no significant difference between all types of vegetation at the depth of $60-80$ and $80-100 \mathrm{~cm}$ during the growing season. For the cropland and shrubland, the volumetric water contents exhibited significant differences at different depths in different months $(P<0.05)$. For the other three vegetation types, in contrast, no significant differences were observed during the entire observation period $(P>0.05)$. Taking cropland as an example, in May, the volumetric water content at the 10-20 and $20-40 \mathrm{~cm}$ levels were significantly greater than at 80 $100 \mathrm{~cm}(P<0.05)$, while there was no significant difference among the $0-20,20-40$ and $40-60 \mathrm{~cm}$ levels $(P>0.05)$. Although the volumetric water content in the $20-40 \mathrm{~cm}$ level was significant higher than the $80-100 \mathrm{~cm}$ level $(P<0.05)$, no significant difference was observed among the 20-40, 4060 and $60-80 \mathrm{~cm}$ levels $(P>0.05)$. The significant difference of the cropland in May was consistent with June. In July, August and September, the volumetric water content in the 10$20 \mathrm{~cm}$ level was significantly greater than the $60-80 \mathrm{~cm}$ and $80-100 \mathrm{~cm}$ levels $(P<0.05)$, whereas no significant difference exhibited between $0-60$ and $20-100 \mathrm{~cm}(P>0.05)$. On the whole, the value of volumetric water content was higher in the grassland and the shrubland sites, whereas the woodland showed lower soil moisture values.

The average soil temperature showed a similar regime among different cover patterns (Fig. 5). The lowest soil temperature was recorded in the shrubland (18 May) while the highest soil temperature was recorded in the cropland (31 July). There was a significant linear correlation between the soil temperature and the atmospheric temperature for each vegetation type during the investigation pe$\operatorname{riod}(P<0.01)$. The correlation coefficient of five vegetation types were 0.76 for the artificial grassland, 0.83 for the crop- land, 0.70 for the shrubland, 0.72 for the woodland, and 0.79 for the native grassland, respectively. Clearly, shrubland and woodland had lower soil temperatures than the other three vegetation types. The mean minimum daily soil temperature of each vegetation type was in May. The mean daily maximum soil temperature was at the end of July or the beginning of August. Although the soil temperature gradually declined in September, the monthly average soil temperature of each vegetation type in September was still higher than in May.

\subsection{Soil moisture evolution with the depth}

In light of the responses of soil moisture variation to the rainfall pulse, stepwise regression was used to analyze the soil volumetric water content profile distributions from 9 to 28 June, 5 to 14 July and from 20 July to 3 August (Fig. 6). At the same time, three typical decreasing periods in these three stages were selected to depict the differences in the water-loss rate of different vegetation types. The slope of regression equation between soil depth and soil moisture indicated that the characteristics of differences in soil volumetric water content were along the soil profile. Regression analysis showed that different vegetation types responded differently to the rainfall events at different periods. For example, from 9 to 28 June, the slope was smallest in the woodland and greatest in the shrubland. Artificial grassland, native grassland and cropland showed an intermediate level, successively. From 5 to 14 July, the slope was also greatest in shrubland, followed by native grassland, woodland and cropland, respectively. Artificial grassland had the smallest slope. From 20 July to 3 August, the slope was smallest in artificial grassland, while greatest in woodland and shrubland. This result indicated that the soil volumetric water content of woodland and shrubland changed dramatically with the increase in soil depth, and the comparison of soil volumetric water content profile distributions revealed that it was easier for water to travel vertically in this stage. Generally speaking, the slope was smaller in cropland and grassland, while shrubland and woodland showed a higher slope, relatively.

Different vegetation types exhibit different daily and cumulative water loss at different periods (Fig. 7). From 12 to 26 June, the beginning of the average soil volumetric moisture content was $0.10 \mathrm{~cm}^{3} \mathrm{~cm}^{-3}$. The native grassland missed the maximum of water, nearly $23.5 \mathrm{~mm}$ in half a month, whereas the woodland lost the minimum of water, only $2.3 \mathrm{~mm}$. In addition, the daily variation of soil moisture was lower and more stable for the woodland than for the other vegetation types, ranging from 0.1 to $0.4 \mathrm{~mm}$. However, the daily soil moisture of the cropland showed the most variability, with a range of variation from 0.89 to $3.0 \mathrm{~mm}$. The cumulative soil water loss of cropland was $20.8 \mathrm{~mm}$, just behind the native grassland. Finally, the artificial grassland and the shrubland exhibited a medium level of loss, with cumulative water loss of 16.2 and $15.5 \mathrm{~mm}$, respectively. In the 

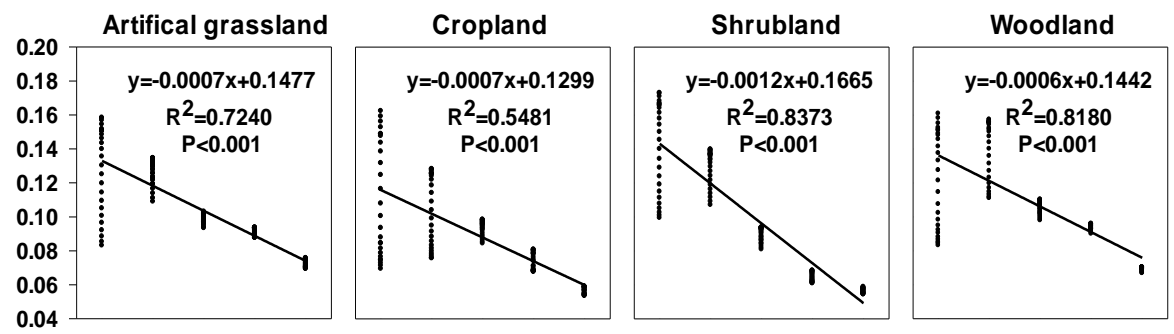

Native grassland
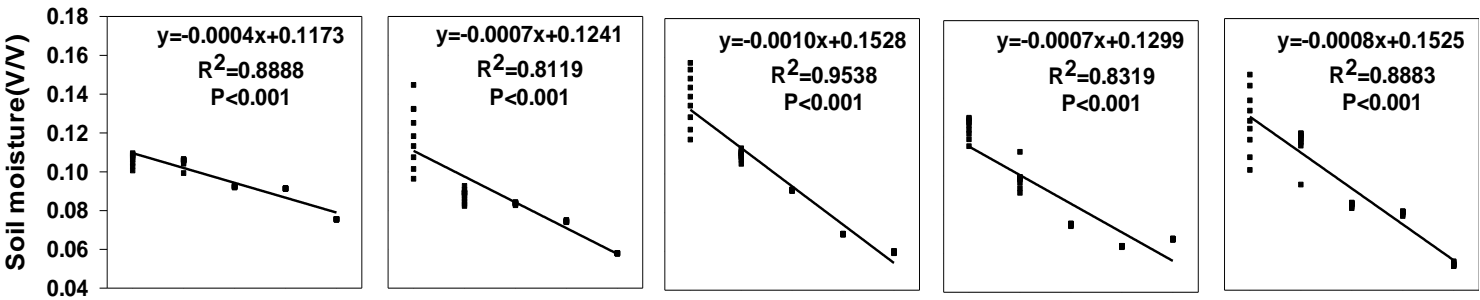

5 July - 14 July
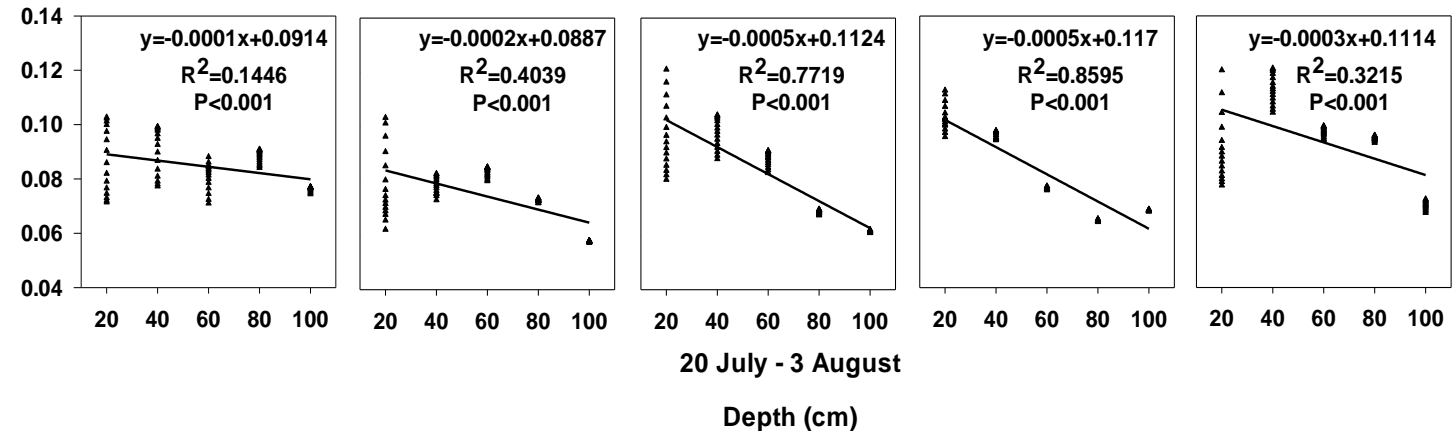

Depth (cm)

Figure 6. Relationship between soil moisture content and soil depth after each rainfall event.

meantime, the daily soil water loss of the artificial grassland showed an increasing trend.

During another two investigated periods (from 7 to 15 July and from 20 July to 1 August), there was a corresponding change of soil water loss for different vegetation types. From 7 to 15 July, the cumulative soil water loss of the artificial grassland was lowest $(2.9 \mathrm{~mm})$, while it was highest for the native grassland and cropland $(10.4 \mathrm{~mm})$. The soil water loss in shrubland was slightly lower than in cropland and the native grassland. The cumulative soil water loss of woodland was lower than the shrubland and higher than the artificial grassland. The daily soil water loss trend of woodland was similar but slightly higher than for the artificial grassland. Meanwhile, cropland and native grassland presented the most variability, ranging from 0.4 to $1.5 \mathrm{~mm}$ and from 0.5 to $1.6 \mathrm{~mm}$, respectively. However, from 20 July to 1 August, the cumulative water loss of the artificial grassland was the highest $(14.6 \mathrm{~mm})$, and the woodland was the lowest $(4.1 \mathrm{~mm})$. The cropland, shrubland and native grassland presented an intermediate level, with average daily losses of $0.85,0.96$ and $1.01 \mathrm{~mm}$, respectively. To summarize, the daily soil water loss trends of shrubland and woodland were more stable than other types during the observation periods, although the cumulative water loss of woodland was relatively lower than in the other types. The daily soil water loss of cropland and native grassland showed more dramatic changes than the other types of vegetation, whereas the cumulative water loss of artificial grassland exhibited strong instability compared with other vegetation types.

\section{Discussion}

\subsection{Effects of rainfall features on soil moisture variation}

The hydrological response of semi-arid ecosystems is mainly controlled by rainfall regimes. Soil moisture was recharged and regulated by precipitation. In our study, an increasing trend in soil moisture appeared after heavy rainfall events, but there were buffer effects following rainfall events based on the groundcover. The results are consistent with the values of Fu et al. (2003), who also conducted studies in a semiarid region of the Loess Plateau. In addition, in semi-arid ecosystems, small rainfall events that cause surface wetness 

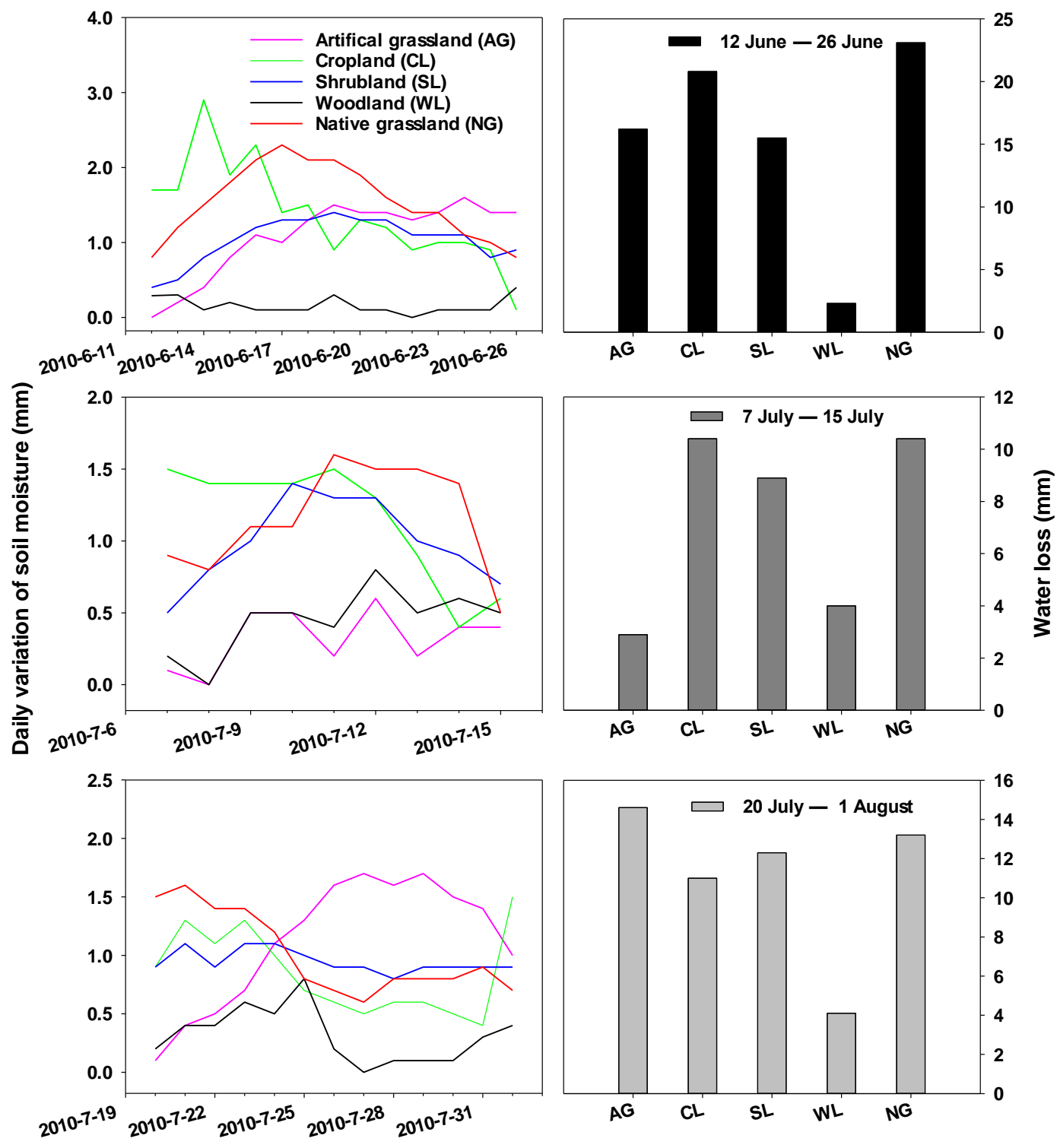

Figure 7. Daily variation of soil moisture and cumulative soil water loss after a rainfall event under five vegetation types.

are more frequent than large events that lead to deeper infiltration (Schwinning and Sala, 2004). The rainfall amount and the number of days between rainfall pulses clearly influenced the soil moisture variation, when events occur in close succession to one another (Loik et al., 2004). The results of the different peak value time of five vegetation types could be explained by the differences in the number of days between rainfall and soil physical properties. Close succession of the rainfall events provided an additive effect. Soil moisture depended strongly on precipitation. Soil moisture variation was similar to the rainfall patterns. For the "impulse type", both of the soil moisture variation and the rainfall regimes are the direct manifestation, particularly in the top soil layer. However, the peak value time at the $20-40$ and $40-60 \mathrm{~cm} \mathrm{lev-}$ els exhibited temporal differences compared with the 60
80 and $80-100 \mathrm{~cm}$ levels. The soil moisture trend began to flatten with increased soil depth. The result indicated that soil water infiltration was different among different vegetation types. In most cases, low bulk density and high porosity can cause higher infiltration rates, which result in a relatively higher soil moisture content at the top soil layers. Meanwhile, it also revealed that different vegetation types have diverse water-use strategies in different periods, based on the different types of response to rainfall pulses. Finally, the characteristic of soil moisture variation suggested that the replenishment of precipitation for the deep layer was limited.

Usually, the root abundance of shrubs and grasses are greater than crops (Jackson et al., 1996), and root decomposition processes change the soil structure and increase rainfall infiltration due to, the channels left after the decompo- 
sition. Therefore, the results showed that grassland exhibits marked trends of soil moisture variation in the deep soil layer, whereas cropland, shrubland and woodland exhibited more stable trends. Five vegetation types showed similar seasonal moisture variation, but there were differences at certain stages. Particularly, soil moisture variation of shrubland and cropland showed significant changes at different soil depths. The fact that all land-use types existed at different water depletion layers is likely related to the diverse root distributions along soil profiles. Moreover, although some water recharge occurred during the growing season, the soil moisture level at the end of September was obviously lower than at the beginning of early May, with the exception of woodland. Soil moisture of woodland at the end of September was close to in early May, indicating that the rainfall pulse had affected less on the soil moisture of the woodland plot. This is partly due to the interception, which would discuss in the next section. Overall, a strong trend of soil water recharge was observed from June to August, whereas only a slight one was observed in September.

\subsection{Soil moisture evolution with the depth}

Soil moisture is depleted over days and weeks primarily by plant uptake, transpiration and soil evaporation. Soil evaporation takes place at the shallow layer with lower root density and varies temporally based on available energy, as reflected in soil temperature values, which themselves lag behind solar radiation inputs (Loik et al., 2004). Therefore, temperature can be considered as one of the key factors leading to soil water loss under high soil moisture content conditions. In our research, artificial grassland, native grassland and cropland exhibited relatively higher soil temperatures, corresponding to greater water loss. However, water loss of artificial grassland showed different trends at different periods. From 5 to 15 July, the smallest regression slope of soil moisture and depth curve were found in artificial grassland compared to other types. This revealed that water more easily traveled vertically in artificial grassland. The amount of infiltration in this process was greater than water loss. At the same time, a relatively higher slope of cropland and native grassland was apparent during the typical decreasing moisture periods, indicating strong interaction between plant uptake and soil moisture as depth increased.

On the other hand, for the native grassland and cropland, poor cover types also led to greater daily water losses via direct evaporation at shallow depth. Soil evaporation varies temporally mainly due to available energy. Hence, the daily water loss of cropland and native grassland showed more dramatic changes during the investigated stages. Furthermore, the temperature at the woodland plot was slightly higher than that of the shrubland. This result is consistent with the conclusion of Wang et al. (2012). However, the amount of water loss was smaller compared with the shrubland, cropland and native grassland, resulted from the interception during the water budget. Crown interception of woodland, clearly, is the first process that diminishes precipitation input to the soil. The foliage has a holding capacity and can intercept all water from small rainfall events, but only a small portion of the water from large rainfall events (Loik et al., 2004; Waring and Running, 2010). During the investigated period, small rainfall events occurred more frequently than heavy rainfall events. The crown interception changed the way and amount in which rainfall came into the soil. Therefore, the smaller the amount of rainfall that infiltrated the woodland, the lower the water loss caused. Throughout the growing season, soil moisture at the end of September was close to that of early May. For the shrubland, the slope of regression equation between soil moisture and depth was the highest. It could be judged that intense interaction occurred between soil moisture and depth, due to the deep root distribution of the shrubland.

\subsection{Implications for vegetation restoration}

Severe scarcity of water resources and land use changes led to the degradation of ecosystem functioning in the semi-arid areas (Cerdà, 1999; Cerdà and Doerr, 2005, 2007; Dickie and Parsons, 2012), also linked to the Loess Plateau of China. Although comprehensive soil and water loss control has achieved some degree of success, it dire challenges remain to be confronted.

As some researchers have previously suggested, different mosaic patterns should be implemented in different area of the Loess Plateau (Fu et al., 2013; She et al., 2010). With the implementation of the Grain for Green project, most sloping croplands were converted to woodland, shrubland and grassland. The most critical experience factor in remedying soil and water erosion is to make the rainfall infiltrate locally. However, due to the crown interception, the amount of rainfall infiltrating woodland is lower than the amount infiltrating shrubland, artificial grassland and native grassland. Therefore, trees were not suitable for large area vegetation restoration. According to Jiang et al. (2013) in terms of pollen records, herbs rather than 11 trees and shrubs should be used for the vegetation restoration programs, but it was inappropriate to select simple vegetation types during the vegetation restoration on a large scale. On the other hand, because of the efficient impediment effects and high survival rate, shrubland was widely adopted in the semi-arid areas, considering the fact that native grassland and artificial grassland can retain more soil moisture. Consequently, our research advocates that a well-matched mosaic vegetation pattern of planting shrub and grass would be appropriate in the study area. Overall, it is imperative that we take drastic measures in the future to research the mechanisms surrounding the influence of community structure characteristics and spatial distribution patterns on soil moisture dynamics, both of the grassland and the shrubland ecosystem of the Loess Plateau. 


\section{Conclusions}

Redistribution of precipitation by different vegetation types probably causes the variability of soil moisture under different rainfall pulses. Soil moisture in the surface layer was significantly affected by precipitation. For the five vegetation types, peak value time of soil moisture appeared after rainfall pulse with a buffer effect, and the trend of the soil moisture began to flatten with increased soil depth. The result showed that soil-water infiltration was different among the different vegetation types, which indicates that different vegetation may have different water-use strategies in different periods. $B D$ values exhibited significant differences among the different soil depths, particularly in the first layer of $0-20 \mathrm{~cm}$. Soil nutrient contents in the surface layer also showed significant higher than the subsoil layer. Compared with other vegetation types, shrubland has the lowest BD and highest SOM. Meanwhile, the characteristic of soil moisture variation suggested that the replenishment of precipitation for the deep layer was limited. During the investigation period, under the influence of interception, smaller amounts of rainfall infiltration occurred under the woodland with lower water loss caused. Shrubland and grassland, however, showed higher soil moisture content. At the same time, the interaction between soil moisture and vegetation has vital implications to optimize vegetation structure and landscape functioning. Our research suggests that vegetation restoration should give priority to shrubland and grassland (both of the artificial and native grassland) in the study region. Hence, further studies should mainly attempt to elucidate the influence of community structure characteristics and spatial distribution patterns on soil moisture dynamics, particularly the effects of fractal features on soil moisture variation, involving both grassland and shrubland ecosystems.

\section{The Supplement related to this article is available online at doi:10.5194/se-6-595-2015-supplement.}

Acknowledgements. We would like to thank Jin Chen, Xiaoping Wang, Xuchun Li, for their generous assistance with the field work. The work was funded by the National Natural Science Foundation of China (41371123, 41390462, 41401209). We thank the editor and two reviewers for their valuable comments and constructive suggestions.

Edited by: P. Pereira

\section{References}

Austin, A. T., Yahdjian, L., Stark, J. M., Belnap, J., Porporato, A., Norton, U., Ravetta, D. A., and Schaeffer, S. M.: Water pulses and biogeochemical cycles in arid and semiarid ecosystems, Oecologia, 141, 221-235, 2004.

Batjes, N. H.: Projected changes in soil organic carbon stocks upon adoption of recommended soil and water conservation practices in the Upper Tana River catchment, Kenya, Land Degrad. Dev., 25, 278-287, 2014.

Bizoza, A. R.: Three-stage analysis of the adoption of soil and water conservation in the highlands of Rwanda, Land Degrad. Dev., 25, 360-372, 2014.

Cerdà, A.: Soil moisture regime under simulated rainfall in a three years abandoned field in southeast Spain, Phys. Chem. Earth, 20, 271-279, 1995.

Cerdà, A.: Seasonal changes of the infiltration rates in a typical Mediterranean Scrubland on limestone in Southeast Spain, J. Hydrol., 198, 198-209, 1997.

Cerdà, A.: Seasonal and spatial variations in infiltration rates in badland surfaces under Mediterranean climatic conditions, Water Resour. Res., 35, 319-328, 1999.

Cerdà, A. and Doerr, S. H.: Soil wettability, runoff and erodibility of major dry-Mediterranean land use types on calcareous soils, Hydrol. Process, 21, 2325-2336, 2007.

Cerdà, A., and Doerr, S. H.: The influence of vegetation recovery on soil hydrology and erodibility following fire: and eleven-year research, Int. J. Wildland Fire, 14, 423-437, 2005.

Chen, H., Shao, M., and Li, Y.: The characteristics of soil water cycle and water balance on steep grassland under natural and simulated rainfall conditions in the Loess Plateau of China, J. Hydrol., 360, 242-251, 2008a.

Chen, H., Shao, M., and Li, Y.: Soil desiccation in the Loess Plateau of China, Geoderma, 143, 91-100, 2008b.

Chen, L., Huang, Z., Gong, J., Fu, B., and Huang, Y.: The effect of land cover/vegetation on soil water dynamic in the hilly area of the loess plateau, China, Catena, 70, 200-208, 2007.

Chen, L., Wang, J., Wei, W., Fu, B., and Wu, D.: Effects of landscape restoration on soil water storage and water use in the Loess Plateau Region, China. Forest. Ecol. Manag., 259, 1291-1298, 2010.

Cornfield, A. H.: Ammonia released on treating soils with $\mathrm{N}$ sodium hydroxide as a possible means of predicting the nitrogensupplying power of soils, Nature (London), 187, 260-261, 1960.

de Melo Carvalho, M. T., de Holanda Nunes Maia, A., Madari, B. E., Bastiaans, L., van Oort, P. A. J., Heinemann, A. B., Soler da Silva, M. A., Petter, F. A., Marimon Jr., B. H., and Meinke, H.: Biochar increases plant-available water in a sandy loam soil under an aerobic rice crop system, Solid Earth, 5, 939-952, doi:10.5194/se-5-939-2014, 2014.

Dickie, J. A. and Parsons, A. J.: Eco-geomorphological processes within grasslands, shrublands and badlands in the simi-arid karoo, South Africa, Land Degrad. Dev., 23, 534-547, 2012.

IUSS-ISRIC-FAO.: World Reference Base for Soil Resources. International Union of Soil Science. International Soil Reference and Information Center, Food and Agriculture Organization of the United Nations, Rome, Italy, 2006.

Fernández-Gálvez, J., Simmonds, L. P., and Barahona, E.: Estimating detailed soil water profile records from point measurements, Eur. J. Soil Sci., 57, 708-718, 2006. 
Fernández-Romero, M. L., Lozano-García, B., and ParrasAlcántara, L.: Topography and land use change effects on the soil organic carbon stock of forest soils in Mediterranean natural areas, Agr. Ecosyst. Environ., 195, 1-9, 2014.

Fu, B., Wang, J., Chen, L., and Qiu, Y.: The effects of land use on soil moisture variation in the Danangou catchment of the Loess Plateau, China, Catena, 54, 197-213, 2003.

Fu, X., Shao, M., Wei, X., Wang, H., and Zeng, C.: Effects of monovegetation restoration types on soil water distribution and balance on a hillslope in Northern Loess Plateau of China, J. Hydrol. Eng., 18, 413-421, 2013.

Gabarrón-Galeote, M. A., Martínez-Murillo, J. F., Quesada, M. A., and Ruiz-Sinoga, J. D.: Seasonal changes in the soil hydrological and erosive response depending on aspect, vegetation type and soil water repellency in different Mediterranean microenvironments, Solid Earth, 4, 497-509, doi:10.5194/se-4-497-2013, 2013.

Gao, X., Wu, P., Zhao, X., Wang, J., and Shi, Y.: Effects of land use on soil moisture variations in a semi-arid catchment: implications for land and agricultural water management, Land Degrad. Dev., 25, 163-172, 2014.

Hewelke, E., Szatylowicz, J., Gnatowski, T., and Oleszczuk, R.: Effects of soil water repellency on soil moisture patterns in a degraded sparic histosol, Land Degrad. Dev., doi:10.1002/ldr.2035, online first, 2014.

Jackson, R., Canadell, J., Ehleringer, J., Mooney, H., Sala, O., and Schulze, E.: A global analysis of root distributions for terrestrial biomes, Oecologia, 108, 389-411, 1996.

Jiang, W., Cheng, Y., Yang, X., and Yang, S.: Chinese Loess Plateau vegetation since the Last Glacial Maximum and its implications for vegetation restoration, J. Appl. Ecol., 50, 440-448, 2013.

Kaleeem Abbasi, M., Mahmood Tahir, M., Sabir, N., and Khurshid, M.: Impact of the addition of different plant residues on nitrogen mineralization-immobilization turnover and carbon content of a soil incubated under laboratory conditions, Solid Earth, 6, 197205, doi:10.5194/se-6-197-2015, 2015.

Koster, R. D., Dirmeyer, P. A., Guo, Z., Bonan, G., Chan, E., Cox, P., Gordon, C., Kanae, S., Kowalczyk, E., and Lawrence, D.: Regions of strong coupling between soil moisture and precipitation, Science, 305, 1138-1140, 2004.

Legates, D. R., Mahmood, R., Levia, D. F., DeLiberty, T. L., Quiring, S. M., Houser, C., and Nelson, F. E.: Soil moisture: A central and unifying theme in physical geography, Prog. Phys. Geog., 35, 65-86, 2011.

Li, Y.: Effects of forest on water circle on the Loess Plateau, J. Nat. Resour., 16, 427-432, 2001. (in Chinese with English abstract).

Littell, R., Milliken, G., Stroup, W., and Wolfinger, R.: SAS system for mixed models, SAS Institute, Cary, NC, USA, 1996.

Liu, G., Jiang, N., Zhang, L., and Liu, Z.: Soil physical and chemical analysis and description of soil profile, Beijing: Standards Press of China, Chinese Standard Press, Beijing, China, 1996 (in Chinese).

Liu, J., Xu, X., Zhang, Y., Tian, Y., and Gao, Q.: Effect of rainfall interannual variability on the biomass and soil water distribution in a semiarid shrub community, Sci. China Life Sci., 53, 729737, 2010.

Liu, Y., Fu, B., Lv, Y., Wang, Z., and Gao, G.: Hydrological responses and soil erosion potential of abandoned cropland in the Loess Plateau, China, Geomorphology, 138, 404-414, 2012.
Loik, M. E., Breshears, D. D., Lauenroth, W. K., and Belnap, J.: A multi-scale perspective of water pulses in dryland ecosystems: climatology and ecohydrology of the western USA, Oecologia, 141, 269-281, 2004.

Lozano-García, B., and Parras-Alcántara, L.: Land use and management effects on carbon and nitrogen in Mediterranean Cambisols, Agr. Ecosyst. Environ., 179, 208-214, 2013.

Lozano-García, B., Parras-Alcántara, L., and del Toro Carrillo de Albornoz, M.: Effects of oil mill wastes on surface soil properties, runoff and soil losses in traditional olive groves in southern Spain, Catena, 85, 187-193, 2011.

Opolot, E., Araya, T., Nyssen, J., Al-Barri, B., Verbist, K., and Cornelis, W. M.: Evaluating in situ water and soil conservation practices with a fully coupled, surface/subsurface processbased hydrological model in Tigray, Ethiopia, Land Degrad. Dev., doi:10.1002/ldr.2335, online first, 2014.

Parras-Alcántara, L. and Lozano-García, B.: Conventional tillage versus organic farming in relation to soil organic carbon stock in olive groves in Mediterranean rangelands (southern Spain), Solid Earth, 5, 299-311, doi:10.5194/se-5-299-2014, 2014.

Porporato, A., D'odorico, P., Laio, F., Ridolfi, L., and RodriguezIturbe, I.: Ecohydrology of water-controlled ecosystems, Adv Water Resour., 25, 1335-1348, 2002.

Qadir, M., Noble, A. D., and Chartres, C.: Adapting to climate change by improving water productivity of soils in dry areas, Land Degrad. Dev., 24, 12-23, 2013.

Qiu, Y., Fu, B., Wang, J., and Chen, L.: Soil moisture variation in relation to topography and land use in a hillslope catchment of the Loess Plateau, China, J. Hydrol., 240, 243-263, 2001.

Sadeghi, S. H. R., Gholami, L., Sharifi, E., Khaledi Darvishan, A., and Homaee, M.: Scale effect on runoff and soil loss control using rice straw mulch under laboratory conditions, Solid Earth, 6, 1-8, doi:10.5194/se-6-1-2015, 2015.

SAS Institute Inc.: The SAS system for windows, SAS Publishing, SAS Institute Cary, NC, 2002.

Santos, J. M., Verheijen, F. G. A., Tavares, W. F., Wahren, A., Feger, K. H., Bernard, J. L., Rial, R. M. E., Keizer, J. J., and Nunes, J. P.: Soil water repellency dynamics in pine and eucalypt plantations in Portugal - a high - resolution time series, Land Degrad. Dev., doi:10.1002/ldr.2251, online first, 2013.

Schwinning, S. and Sala, O. E.: Hierarchy of responses to resource pulses in arid and semi-arid ecosystems, Oecologia, 141, 211220, 2004.

Shangguan, Z. P. and Zheng, S.: Ecological properties of soil water and effects on forest vegetation in the Loess Plateau, Int. J. Sust. Dev. World, 13, 307-314, 2006.

She, D., Shao, M., Timm, L., Sentís, I. P., Reichardt, K., and Yu, S.: Impacts of land-use pattern on soil water-content variability on the Loess Plateau of China, Acta Agr. Scand. B-S. P., 60, 369380, 2010.

Shi, H. and Shao, M.: Soil and water loss from the Loess Plateau in China, J. Arid Environ., 45, 9-20, 2000.

Wang, L., Shao, M., Wang, Q., Jia, Z., and Li, J.: Review of research on soil desiccation in the Loess Plateau, T. CSAE., 20, 27-31, 2004 (in Chinese with English Abstract).

Wang, S., Fu, B. J., Gao, G. Y., Yao, X. L., and Zhou, J.: Soil moisture and evapotranspiration of different land cover types in the Loess Plateau, China, Hydrol. Earth Syst. Sci., 16, 2883-2892, doi:10.5194/hess-16-2883-2012, 2012. 
Wang, Y., Shao, M., Zhu, Y., and Liu, Z. P.: Impacts of land use and plant characteristics on dried soil layers in different climatic regions on the Loess Plateau of China, Agr. Forest Meteorol., 151, 437-448, 2011.

Waring, R. H. and Running, S. W.: Forest ecosystems: analysis at multiple scales, 3rd Edn., Elsevier Academic Press, UK, 2010.

Wei, W., Chen, L., Fu, B., Huang, Z., Wu, D., and Gui, L.: The effect of land use and rainfall regimes on runoff and erosion in the loess hilly area, China. J. Hydrol., 335, 247-258, 2007.

Wei, W., Jia, F., Yang, L., Chen, L., Zhang, H., and Yang, Y.: Effects of surficial condition and rainfall intensity on runoff in a loess hilly area, China, J. Hydrol., 513, 115-126, 2014.

Wei, Y., Guo, K., and Chen, J.: Effect of precipitation pattern on recruitment of soil water in KUBUQI desert, northwestern China, J. Plant Ecol., 6, 1346-1355, 2008.

Weltzin, J. F. and Tissue, D. T.: Resource pulses in arid environments-patterns of rain, patterns of life, New Phytol., 157, 171-173, 2003.

Wu, Y., Xu, G., and Shao, H. B.: Furfural and its biochar improve the general properties of a saline soil, Solid Earth, 5, 665-671, doi:10.5194/se-5-665-2014, 2014.
Yang, L., Wei, W., Chen, L., and Mo, B., Response of deep soil moisture to land use and afforestation in the semi-arid Loess Plateau, China, J. Hydrol., 475, 111-122, 2012.

Yang, L., Wei, W., Chen, L., Chen, W., and Wang, J.: Response of temopral variation of soil moisture to vegetation restoration in semi-arid Loess Plateau, China, Catena, 115, 123-133, 2014.

Ziadat, F. M. and Taimeh, A. Y.: Effect of rainfall intensity, slope, land use and antecedent soil moisture on soil erosion in an arid environment, Land Degrad. Dev., 24, 582-590, doi:10.1002/ldr.2239, 2013.

Zhao, G., Mu, X., Wen, Z., Wang, F., and Gao, P.: Soil erosion, conservation, and Eco-environment changes in the Loess Plateau of China, Land Degrad. Dev., 24, 499-510, doi:10.1002/ldr.2246, 2013.

Zhu, H., Shi, Z., Fang, N., Wu, L., Guo, Z., and Yang, Y.: Soil moisture response to environmental factors following precipitation events in a small catchment, Catena, 120, 73-80, 2014. 